\title{
Pattern Recognition for Defect Detection in Uncontrolled Environment Railway Applications
}

\author{
Giuseppe Di Leo ${ }^{1}$, Roald Lengu ${ }^{2}$, Nadia Mazzino ${ }^{2}$, and Alfredo Paolillo ${ }^{1}$ \\ ${ }^{1}$ Dept. of Industrial Engineering, University of Salerno, Italy \\ apaolillo@unisa.it \\ ${ }^{2}$ Ansaldo STS, Genoa, Italy \\ roald. lengu@ansaldo-sts.com
}

\begin{abstract}
The rise in prominence of safety and maintenance cost saving related issues in railway systems is becoming more and more an important driver in the design and deployment of sophisticated Wayside Train Monitoring Systems (WTMS). In the last 20 years computer vision based WTMS have evolved from simple Hot Axle Bearing Detectors (HABD) to sophisticated Video Monitoring Systems (VMS).
\end{abstract}

\section{Introduction}

The aim of this paper is to present a Video Monitoring System, developed by Ansaldo STS with the support of University of Salerno, for pattern recognition, aiming at automatic defect detection on transiting rolling stocks. The main challenges presented by such a system are related to:

- use of line scan cameras acquiring high speed moving objects, which present a different image projection system and several image quality problems compared to standard area scan cameras acquiring still objects;

- an uncontrolled illuminated environment, raising image acquisition quality problems;

- the definition of a pattern recognition and defect detection approach on rolling stock interested components.

\section{Imaging with Line Scan Cameras}

The geometry for the image formation is described in Fig. 1, where the lateral surface of a transiting stock is imaged by a line scan camera and is lit by an illuminator array made of a number of illuminator units. The sensor array is oriented vertically. Among the different possible choices, the world reference frame has been placed onto the lateral surface, with the $z$ axis oriented as the forward motion direction, and the $y$ axis vertically oriented. The horizontal camera is positioned at a horizontal distance $x_{\text {cam }}$ from the lateral surface, while the illuminator unit is placed at a horizontal distance $x_{\text {led }}$ from the same surface. 


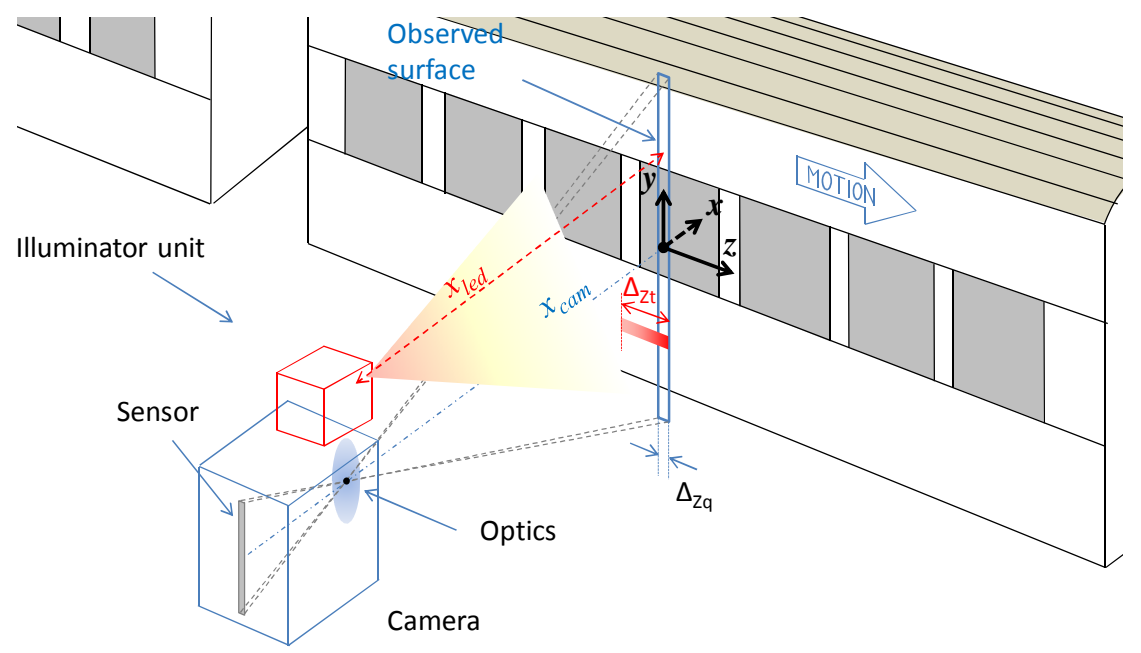

Fig. 1. Geometry for camera, object and illuminator unit

The line scan camera can grab images made by a single line of pixels, or by very few lines of pixels, with a quite high rate, of the order of tens of thousands lines per second. The image formation is obtained by stacking the lines grabbed during the transit of the object (the train) onto a single wide image. This means that the relationship between the train speed and the line per second acquisition rate is fundamental in determining the horizontal scale of the image in terms of pixel $/ \mathrm{m}$. The horizontal resolution can be expressed as a combination of a contribution $\Delta_{z q}$ due to the sensor cell size, which covers a finite size horizontal length on the object surface according to the perspective, and a contribution $\Delta_{z t}$ due to the movement of the object during the sensor integration time, as shown in Fig. 1. On the other hand, the image formation along the vertical direction can be modeled with the traditional perspective relationships, commonly referred to as the camera pin-hole model [1]. The geometry of the acquisition system and of the object lets simplify the description of the problem which can be studied as a two-dimensional one rather than three-dimensional. With reference to Fig. 2, where a camera, slanted by a $\beta_{\text {cam }}$ tilt angle, frames a part of the illuminated surface, the relationship between the pixel coordinate $v$ and the world coordinate $y$ on the object surface reduces to:

$$
\frac{y}{x_{c a m}}=\operatorname{tg}\left(\beta_{c a m}-\operatorname{tg}^{-1}\left(\frac{\left(v_{0}-v\right) s_{v}}{f}\right)\right)
$$

where $f$ is the camera constant, i.e. the distance between the sensor and the projection center of the lens, $s_{v}$ is vertical size of the sensor cell and $v_{0}$ is the camera principal point, i.e. the pixel coordinate of the cell where the optical axis intersects the sensor plane. The system is also composed of an illumination sub-system based on an array of LEDs, which allows to reach an adequate image contrast even in different environmental illumination conditions [2-4]. 


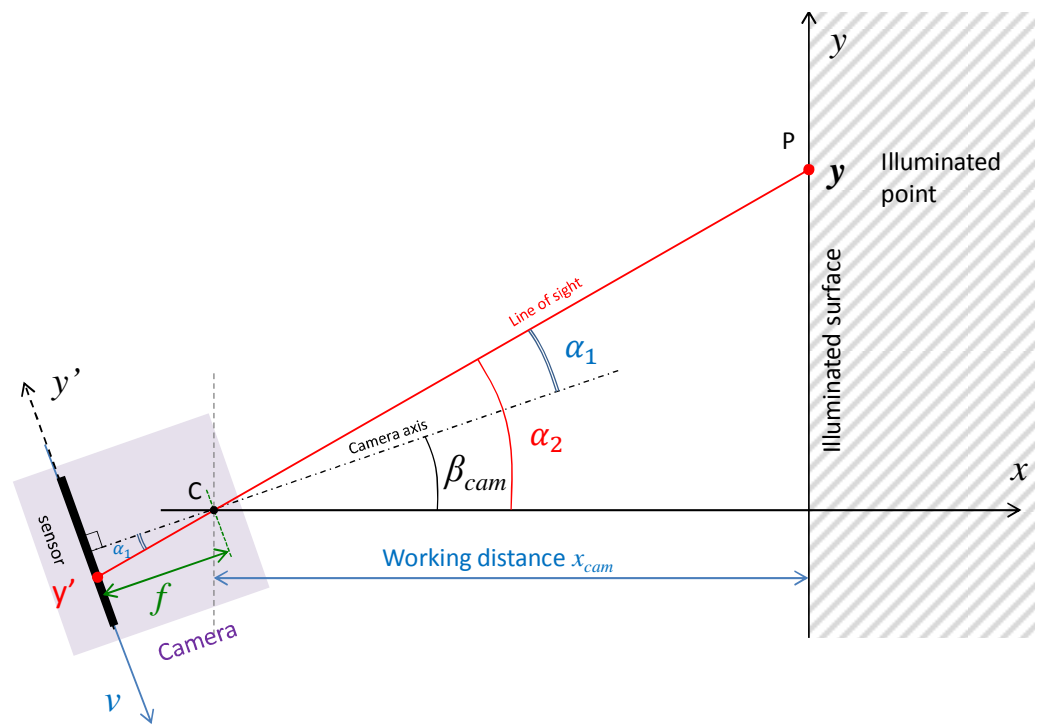

Fig. 2. Image formation geometry

\section{Pattern Recognition Approach for Defect Detection}

The software for the defect detection is implemented as a set of classes having methods aiming at the application of specific localization, analysis and fine segmentation algorithms onto the image, which will be detailed in the full paper. The output of this module is a series of values, specific for each part to be inspected, giving the position of the object within the image, the measurement of its characteristic value and possible other values, e.g. for displaying the results onto the image.

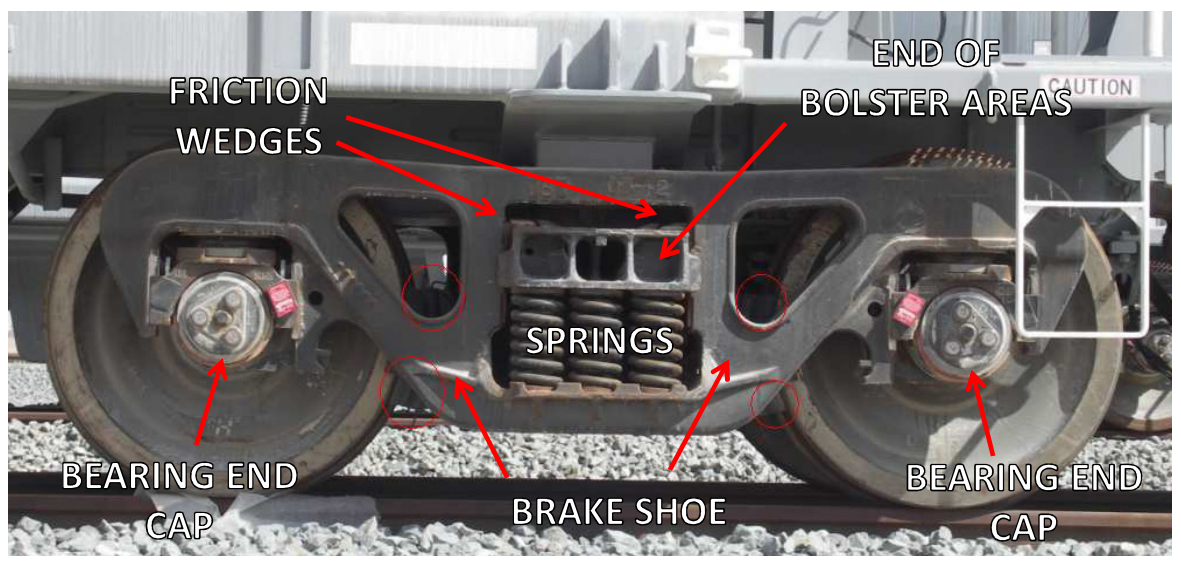

Fig. 3. Examples of parts to be recognized and inspected by the VMS 


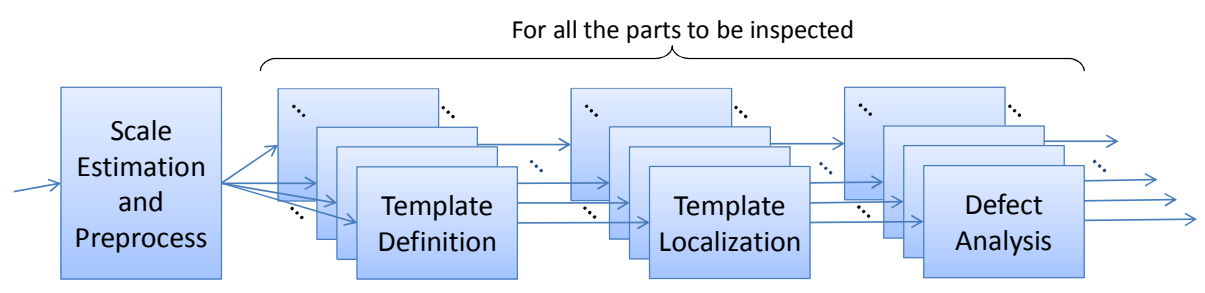

Fig. 4. Operation of the defect detection module

In Fig. 3, some examples of objects to be inspected are shown, which are mainly related to specific mechanical parts of the bogie and of the parts coupling subsequent rolling stocks. The operation of the defect detection module is sketched in Fig. 4. At first, a module retrieves basic information about a relative scale of the image. Other pre-processing tasks are run at this point, such as image smoothing or contrast enhancement. This is necessary for the proper sizing of the templates that are to be built in the following modules, since the same software should adaptively process images taken from different distances, e.g. in case the rolling stocks move on two or more tracks. The subsequent processing path is composed of a set of functionally similar tasks, each one run for each part to be inspected. A single tasks begins with the definition of a template that matches the part to be inspected, then the template is localized with a pattern matching approach [5], and finally specific analysis tasks aiming at measuring the required values.

\section{$4 \quad$ Results}

An example of processing is reported in Fig. 5. A template (Fig.5a) is defined as a combination of geometrical entities, such as circle arcs and line segments. This template is characteristic of a specific feature to be inspected: the bearing end caps. The occurrences of this template are located within the image with pattern matching functions able to locate them even under rotation, shift and limited scale variation.

a)
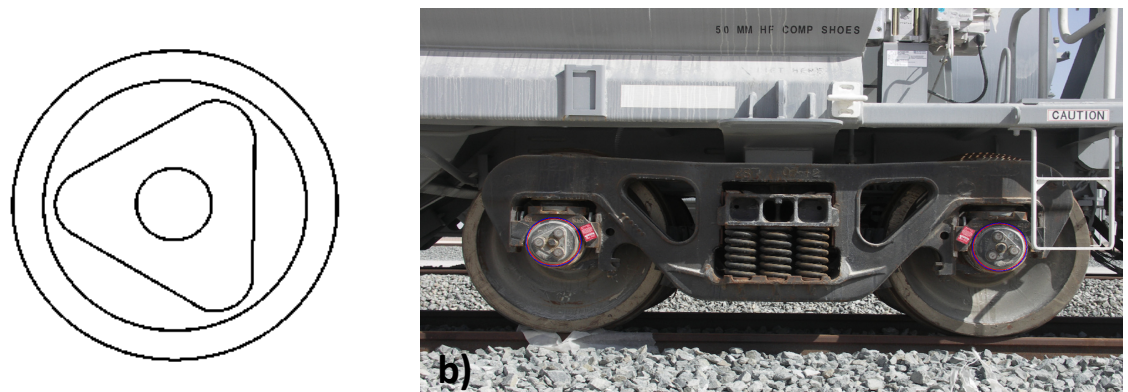

Fig. 5. Example of pattern matching: a) template; b) localization of the template within the image in order to locate the bearing end caps 


\section{Conclusions}

A Video Monitoring System has been introduced aiming at automatic defect detection on transiting rolling stocks. The imaging hardware is based on line scan cameras for the acquisition of images of moving objects. It applies pattern matching techniques in order to locate specific features to be inspected. The full paper will present details of the dimensioning of the acquisition system and additional results.

\section{References}

1. Hartley, R., Zisserman, A.: Multiple View Geometry in Computer Vision, 2nd edn. Cambridge University Press

2. Forsyth, D.A., Ponce, J.: Computer Vision: A Modern Approach. Prentice Hall (2002)

3. Handbook of Optics, 3rd edn., vol. 2. McGraw-Hill (2010)

4. Nayar, S.K., Ikeuchi, K., Kanade, T.: Surface Reflection: Physical and Geometrical Perspectives. IEEE Transactions on Pattern Analysis and Machine Intelligence 13(7), 611-634 (1991)

5. Gonzalez, R.C., Woods, R.C.: Digital Image Processing, 2nd edn. Prentice Hall, Inc. (2002) 